\title{
An oscillation criterion for half-linear second order differential equations
}

\author{
Jana Rezničková
}




\title{
AN OSCILLATION CRITERION FOR HALF-LINEAR SECOND ORDER DIFFERENTIAL EQUATIONS
}

\author{
JANA ŘEZNÍČKOVÁ
}

[Received: November 12, 2003]

\author{
Aвstract. We investigate oscillatory properties of the half-linear second order dif- \\ ferential equation$$
\left(\Phi\left(x^{\prime}\right)\right)^{\prime}+c(t) \Phi(x)=0
$$ \\ where $\Phi(x):=|x|^{p-2} x$. This equation is regarded as a perturbation of the generalized \\ Euler equation \\ where $\gamma_{p}=\left(\frac{p-1}{p}\right)^{p}$.

$$
\left(\Phi\left(x^{\prime}\right)\right)^{\prime}+\frac{\gamma_{p}}{t^{p}} \Phi(x)=0,
$$ \\ Mathematics Subject Classification: 34C10
}

Keywords: Half-linear equation, variational principle, oscillation

\section{InTRODUCTION}

In this paper, we investigate oscillatory properties of the half-linear second order differential equation

$$
\left(\Phi\left(x^{\prime}\right)\right)^{\prime}+c(t) \Phi(x)=0,
$$

where $\Phi(x):=|x|^{p-2} x, p>1, t \in I:=[T, \infty)$, and $c$ is a real-valued continuous function.

Oscillation theory of (1) attracted considerable attention in the past years and it was shown that many of the oscillation criteria for the linear Sturm-Liouville second order differential equation

$$
x^{\prime \prime}+c(t) x=0
$$

(which is the special case $p=2$ of (1)) can be extended to (1).

In these criteria, equation (1) is essentially viewed as a perturbation of the oneterm (nonoscillatory) equation

$$
\left(\Phi\left(x^{\prime}\right)\right)^{\prime}=0
$$

and the nonoscillation or oscillation of (1) is "measured" by the "smallness" of the function $c$.

Supported by Grant 201/01/0079 of the Czech Grant Agency. 
In this paper we investigate equation (1) not as a perturbation of (3), but as a perturbation of the generalized Euler equation

$$
\left(\Phi\left(x^{\prime}\right)\right)^{\prime}+\frac{\gamma_{p}}{t^{p}} \Phi(x)=0
$$

where $\gamma_{p}=\left(\frac{p-1}{p}\right)^{p}$ is the so-called critical constant in this equation. In particular, this equation is nonoscillatory for $\gamma \leq \gamma_{p}$ and oscillatory for $\gamma>\gamma_{p}$, see [3]. A similar idea was used in $[\mathbf{2 , 4 , 5}$. In these papers, nonoscillation criteria were mostly based on the Riccati technique consisting in the fact that if $x$ is a nonzero solution of (1) then

solves the generalized Riccati equation

$$
w(t)=\frac{\Phi\left(x^{\prime}(t)\right)}{\Phi(x(t))}
$$

$$
w^{\prime}+c(t)+(p-1)|w|^{q}=0
$$

where $q$ is the conjugate number to $p$, i. e., $\frac{1}{p}+\frac{1}{q}=1$.

Here we use the variational principle which is based on the relationship between disconjugacy of $(1)$ in $[a, b]$ and the positivity of the " $p$-degree" functional

$$
\mathscr{F}(y ; a, b)=\int_{a}^{b}\left[\left|y^{\prime}\right|^{p}-c(t)|y|^{p}\right] d t
$$

in the class of (sufficiently smooth) functions satisfying $y(a)=0=y(b)$. In particular, equation (1) is disconjugate in $[a, b]$, i. e., any nontrivial solution has at most one zero in $[a, b])$ if and only if $\mathscr{F}(y ; a, b)>0$ for every nontrivial function $y \in W^{1, p}(a, b)$ with $y(a)=0=y(b)$. More details will be given in the next section. The variational approach was used, e. g., in [6].

The main result of this paper is the oscillation criterion which answers in the affirmative way the conjecture posed in [2].

This paper is organized as follows. In the Section 2, we recall the properties of equation (4) needed for our investigation. We also formulate some auxiliary results and give known results. In Section 3, we present the main result of the paper, an oscillation criterion for (1). Section 4 contains some remarks about possible extensions of the results of this paper.

\section{Preliminaries}

First we return to Euler equation (4). In the critical case $\gamma=\gamma_{p}$, this equation possesses the solution $\tilde{x}(t)=t^{\frac{p-1}{p}}$ and any linearly independent solution satisfies the relation

$$
x(t) \sim t^{\frac{p-1}{p}} \lg ^{\frac{2}{p}} t,
$$

where $f_{1} \sim f_{2}$ for a pair of functions $f_{1}, f_{2}$ means that $L:=\lim _{t \rightarrow \infty} \frac{f_{1}(t)}{f_{2}(t)}$ exists and $0<|L|<\infty$. 
Now we formulate exactly the relationship between the positivity of the functional $\mathscr{F}$ given in (5) and diconjugacy of (1). This statement is proved, e. g., in [6] and we formulate it here in a slightly modified form as needed in the next section.

Lemma 1. Equation (1) is oscillatory if and only if for every $T \in \mathbb{R}$ there exists $0 \not \equiv \in W_{0}^{1, p}(T, \infty)$ such that

$$
\mathscr{F}(y ; T, \infty)=\int_{T}^{\infty}\left[\left|y^{\prime}(t)\right|^{p}-c(t)|y(t)|^{p}\right] d t \leq 0 .
$$

The following lemma sumarizes the results of Došlý and Lomtatidze [2] where (1) is viewed as a perturbation of the Euler equation (4).

Lemma 2. The following statements hold.

(i) Suppose that $c(t) \geq 0$ and

$$
\liminf _{t \rightarrow \infty} \lg t \int_{t}^{\infty}\left(c(s)-\frac{\gamma_{p}}{s^{p}}\right) s^{p-1} d s>\frac{1}{2}\left(\frac{p-1}{p}\right)^{p-1} .
$$

Then (1) is oscillatory.

(ii) If

$$
\limsup _{t \rightarrow \infty} \lg t \int_{t}^{\infty}\left(c(s)-\frac{\gamma_{p}}{s^{p}}\right) s^{p-1} d s<\frac{1}{2}\left(\frac{p-1}{p}\right)^{p-1}
$$

and

$$
\liminf _{t \rightarrow \infty} \lg t \int_{t}^{\infty}\left(c(s)-\frac{\gamma_{p}}{s^{p}}\right) s^{p-1} d s>-\frac{3}{2}\left(\frac{p-1}{p}\right)^{p-1},
$$

then (1) is nonoscillatory.

Recall also the results of the paper by Elbert and Schneider based on the connection of oscillatory behaviour between the half-linear differential equation

$$
\left(\Phi\left(x^{\prime}\right)\right)^{\prime}+\left(\frac{p-1}{p}\right)^{p} \frac{1}{t^{p}}\left[1+\frac{p}{2(p-1)} \delta(t)\right] \Phi(x)=0
$$

(where $\delta(t)$ is a piecewise continuous function on $\left(t_{0}, \infty\right)$, for some $t_{0} \geq 0$ ) and the linear differential equation

$$
\left(t z^{\prime}\right)^{\prime}+\frac{\delta(t)}{t} z=0
$$

Lemma 3. Suppose that $\int^{\infty} \frac{\delta(t)}{t} d t$ is convergent and $\int_{t}^{\infty} \frac{\delta(s)}{s} d s \geq 0$ for large $t$.

(i) If $p \geq 2$ and the linear differential equation (10) is nonoscillatory, then the half-linear differential equation (9) is also nonoscillatory.

(ii) If $p \in(1,2]$ and equation (9) is nonoscillatory, then equation (10) is also nonoscillatory. 
Note that the equivalence of nonoscillation of (9) and (10) is an open problem. However, under some restrictions on the function $\delta(t)$ or on the corresponding linear differential equation (10), one can establish a closer connection between (9) and (10). For more details, see [5].

\section{OsCillation CRITERION}

In this section, we present the main result of the paper - an oscillation criterion for (1). This equation is written in the form

$$
\left(\Phi\left(x^{\prime}\right)\right)^{\prime}+\frac{\gamma_{p}}{t^{p}} \Phi(x)+\left(c(t)-\frac{\gamma_{p}}{t^{p}}\right) \Phi(x)=0
$$

and it is viewed as a perturbation of the generalized Euler equation (4).

Theorem 1. Suppose that

$$
\liminf _{t \rightarrow \infty} \frac{1}{\lg t} \int_{1}^{t}\left(c(s)-\frac{\gamma_{p}}{s^{p}}\right) s^{p-1} \lg ^{2} s d s>2\left(\frac{p-1}{p}\right)^{p-1} .
$$

Then (1) is oscillatory.

Proof. According to Lemma 1, it suffices to find, for any $T \in \mathbb{R}$, a function $y \in$ $W^{1, p}(t, \infty)$ with a compact support in $(T, \infty)$, say $\left[t_{0}, t_{3}\right]$, such that the functional $\mathscr{F}$ given by (5) is negative. To this end, let $T \in \mathbb{R}$ be arbitrary and $T<t_{0}<t_{1}<t_{2}<$ $t_{3}$. Further, let $f$ be arbitrary sufficiently smooth function satisfying the boundary conditions

and let

$$
f\left(t_{0}\right)=0, \quad f\left(t_{1}\right)=h\left(t_{1}\right)
$$

$$
g(t)=h\left(t_{2}\right) \frac{t^{\frac{p-1}{p}} \lg ^{\frac{2}{p}} \frac{t_{3}}{t}}{t_{2}^{\frac{p-1}{p}} \lg ^{\frac{2}{p}} \frac{t_{3}}{t_{2}}}
$$

where $h(t)=t^{\frac{p-1}{p}} \lg ^{\frac{2}{p}} t$. Define a test function $y$ as follows:

$$
y(t)=\left\{\begin{array}{lll}
0 & \text { if } & T \leq t \leq t_{0} \\
f(t) & \text { if } & t_{0} \leq t \leq t_{1} \\
h(t) & \text { if } & t_{1} \leq t \leq t_{2} \\
g(t) & \text { if } & t_{2} \leq t \leq t_{3} \\
0 & \text { if } & t \geq t_{3}
\end{array}\right.
$$

For the computation to follow, we put

$$
\hat{g}(t)=t^{\frac{p-1}{p}} \lg ^{\frac{2}{p}} \frac{t_{3}}{t}
$$

and

$$
K\left(t_{2}, t_{3}\right)=t_{2}^{\frac{p-1}{p}} \lg ^{\frac{2}{p}} \frac{t_{3}}{t_{2}} .
$$


Then, the function $g$ can be written in the form

$$
g(t)=\frac{h\left(t_{2}\right)}{K\left(t_{2}, t_{3}\right)} \hat{g}(t) .
$$

Further, we set

$$
\begin{aligned}
L=\mathscr{F}\left(f ; t_{0}, t_{1}\right) & =\int_{t_{0}}^{t_{1}}\left[\left|f^{\prime}(t)\right|^{p}-\frac{\gamma_{p}}{t^{p}}|f(t)|^{p}\right] d t-\int_{t_{0}}^{t_{1}}\left[c(t)-\frac{\gamma_{p}}{t^{p}}\right]|f(t)|^{p} d t, \\
G\left(h ; t_{1}, t_{2}\right) & =\int_{t_{1}}^{t_{2}}\left[\left|h^{\prime}(t)\right|^{p}-\frac{\gamma_{p}}{t^{p}}|h(t)|^{p}\right] d t, \\
G\left(g ; t_{2}, t_{3}\right) & =\int_{t_{2}}^{t_{3}}\left[\left|g^{\prime}(t)\right|^{p}-\frac{\gamma_{p}}{t^{p}}|g(t)|^{p}\right] d t \\
H\left(h ; t_{1}, t_{2}\right) & =\int_{t_{1}}^{t_{2}}\left[c(t)-\frac{\gamma_{p}}{t^{p}}\right]|h(t)|^{p} d t \\
H\left(g ; t_{2}, t_{3}\right) & =\int_{t_{2}}^{t_{3}}\left[c(t)-\frac{\gamma_{p}}{t^{p}}\right]|g(t)|^{p} d t \\
2 & =\int_{t_{2}}^{t_{3}}\left[c(t)-\frac{\gamma_{p}}{t^{p}}\right]|h(t)|^{p}\left|\frac{g(t)}{h(t)}\right|^{p} d t .
\end{aligned}
$$

Then we have

$$
\begin{aligned}
\mathscr{F}\left(y ; t_{0}, t_{3}\right) & =\int_{t_{0}}^{t_{3}}\left[\left|y^{\prime}(t)\right|^{p}-c(t)|y(t)|^{p}\right] d t \\
& =\int_{t_{0}}^{t_{3}}\left[\left|y^{\prime}(t)\right|^{p}-\frac{\gamma_{p}}{t^{p}}|y(t)|^{p}\right] d t-\left.\int_{t_{0}}^{t_{3}}\left[c(t)-\frac{\gamma_{p}}{t^{p}}\right] y(t)\right|^{p} d t \\
& \left.=\left.\int_{t_{0}}^{t_{1}}\left[\left|f^{\prime}(t)\right|^{p}-\frac{\gamma_{p}}{t^{p}} t\right)\right|^{p}\right] d t-\int_{t_{0}}^{t_{1}}\left[c(t)-\frac{\gamma_{p}}{t^{p}}\right]|f(t)|^{p} d t \\
& +\int_{t_{1}}^{t_{2}}\left[\left|h^{\prime}(t)\right|^{p}-\frac{\gamma_{p}}{t^{p}}|h(t)|^{p}\right] d t+\int_{t_{2}}^{t_{3}}\left[\left|g^{\prime}(t)\right|^{p}-\frac{\gamma_{p}}{t^{p}}|g(t)|^{p}\right] d t \\
& -\int_{t_{1}}^{t_{2}}\left[c(t)-\frac{\gamma_{p}}{t^{p}}\right]|h(t)|^{p} d t-\int_{t_{2}}^{t_{3}}\left[c(t)-\frac{\gamma_{p}}{t^{p}}\right]|g(t)|^{p} d t \\
& =\mathscr{F}\left(f ; t_{0}, t_{1}\right)+G\left(h ; t_{1}, t_{2}\right)+G\left(g ; t_{2}, t_{3}\right)-H\left(h ; t_{1}, t_{2}\right)-H\left(g ; t_{2}, t_{3}\right) .
\end{aligned}
$$

Let us start with computing $G\left(h ; t_{1}, t_{2}\right)$. Since

$$
h^{\prime}(t)=\frac{p-1}{p} t^{-\frac{1}{p}} \lg ^{\frac{2}{p}} t\left(1+\frac{2}{(p-1) \lg t}\right),
$$

then, using the asymptotic formula

$$
(1+x)^{p}=1+p x+\frac{1}{2} p(p-1) x^{2}+o\left(x^{2}\right) \quad \text { as } x \rightarrow 0,
$$


we get

$$
\left|h^{\prime}(t)\right|^{p}=\left(\frac{p-1}{p}\right)^{p} t^{-1} \lg ^{2} t\left[1+\frac{2 p}{(p-1) \lg t}+\frac{2 p}{(p-1) \lg ^{2} t}+o\left(\frac{1}{\lg ^{2} t}\right)\right] .
$$

Hence,

$$
\begin{aligned}
G\left(h ; t_{1}, t_{2}\right) & =\int_{t_{1}}^{t_{2}}\left[\left|h^{\prime}(t)\right|^{p}-\frac{\gamma_{p}}{t^{p}}|h(t)|^{p}\right] d t \\
& =\int_{t_{1}}^{t_{2}}\left[\left(\frac{p-1}{p}\right)^{p} t^{-1} \lg ^{2} t\left\{1+\frac{2 p}{(p-1) \lg t}+\frac{2 p}{(p-1) \lg ^{2} t}+o\left(\frac{1}{\lg ^{2} t}\right)\right\}\right. \\
& \left.-\frac{1}{t^{p}}\left(\frac{p-1}{p}\right)^{p} t^{p-1} \lg ^{2} t\right] d t \\
& =2\left(\frac{p-1}{p}\right)^{p-1} \int_{t_{1}}^{t_{2}}\left[\frac{1}{t} \lg t+\frac{1}{t}+o\left(\frac{1}{t}\right)\right] d t \\
& =2\left(\frac{p-1}{p}\right)^{p-1}\left[\left.\lg t\right|_{t_{1}} ^{t_{2}}+\left.\frac{1}{2} \lg ^{2} t\right|_{t_{1}} ^{t_{2}}+o\left(\lg t_{2}\right)\right] \\
& =\left.2\left(\frac{p-1}{p}\right)^{p-1} \lg t\right|_{t_{1}} ^{t_{2}}+\left.\left(\frac{p-1}{p}\right)^{p-1} \lg ^{2} t\right|_{t_{1}} ^{t_{2}}+o\left(\lg t_{2}\right)
\end{aligned}
$$

as $t_{2} \rightarrow \infty$. Concerning $G\left(h ; t_{1}, t_{2}\right)$, we obtain

$$
\begin{aligned}
G\left(g ; t_{2}, t_{3}\right) & =\frac{h^{p}\left(t_{2}\right)}{K^{p}\left(t_{2}, t_{3}\right)} \int_{t_{2}}^{t_{3}}\left[\left|\hat{g}^{\prime}(t)\right|^{p}-\frac{\gamma_{p}}{t^{p}}|\hat{g}(t)|^{p}\right] d t \\
& =\frac{\lg ^{2} t_{2}}{\lg ^{2} \frac{t_{3}}{t_{2}}}\left(\frac{p-1}{p}\right)^{p} \int_{t_{2}}^{t_{3}}\left\{\frac{1}{t} \lg ^{2} \frac{t_{3}}{t}\left(1-\frac{2}{(p-1) \lg t}\right)^{p}-\frac{1}{t} \lg ^{2} \frac{t_{3}}{t}\right\} d t \\
& =2\left(\frac{p-1}{p}\right)^{p-1} \frac{\lg ^{2} t_{2}}{\left.\lg ^{2} t\right|_{t_{2}} ^{t_{3}}}\left[\left.\lg t\right|_{t_{2}} ^{t_{3}}-\left.\lg t_{3} \lg t\right|_{t_{2}} ^{t_{3}}+\left.\frac{1}{2} \lg ^{2} t\right|_{t_{2}} ^{t_{3}}+o\left(\lg t_{3}\right)\right] .
\end{aligned}
$$

In the last line of the previous computation we used again the formula (13). Now, let $t_{2}$ be fixed for a moment. We will show that

$$
\lim _{t_{3} \rightarrow \infty} G\left(g ; t_{2}, t_{3}\right)=-\left(\frac{p-1}{p}\right)^{p-1} \lg ^{2} t_{2}
$$


From (14), we have

$$
\begin{gathered}
\frac{\left.\lg t\right|_{t_{2}} ^{t_{3}}-\left.\lg t_{3} \lg t\right|_{t_{2}} ^{t_{3}}+\left.\frac{1}{2} \lg ^{2} t\right|_{t_{2}} ^{t_{3}}+o\left(\lg t_{3}\right)}{\left.\lg ^{2} t\right|_{t_{2}} ^{t_{3}}} \\
=\frac{\lg t_{3}-\lg t_{2}-\lg t_{3}\left(\lg t_{3}-\lg t_{2}\right)+\frac{1}{2} \lg ^{2} t_{3}-\frac{1}{2} \lg ^{2} t_{2}+o\left(\lg t_{3}\right)}{\lg ^{2} t_{3}-\lg ^{2} t_{2}} \\
=\frac{-\frac{1}{2} \lg ^{2} t_{3}+\lg t_{3}-\lg t_{2}+\lg t_{3} \lg t_{2}-\frac{1}{2} \lg ^{2} t_{2}+o\left(\lg t_{3}\right)}{\lg ^{2} t_{3}-\lg ^{2} t_{2}} \\
=\frac{-\frac{1}{2}+\frac{1}{\lg _{3} t_{3}}-\frac{\lg _{2}}{\lg ^{2} t_{3}}+\frac{\lg t_{2}}{\lg _{3}}-\frac{1}{2} \frac{\lg ^{2} t_{2}}{\lg ^{2} t_{3}}+o\left(\frac{1}{\lg _{3}}\right)}{1-\frac{\lg ^{2} t_{2}}{\lg ^{2} t_{3}}} \longrightarrow-\frac{1}{2}
\end{gathered}
$$

as $t_{3} \rightarrow \infty$ and, thus, (15) holds indeed. In the next part of the proof, we show that the function $\frac{g}{h}$ is strictly monotonic on $\left(t_{2}, t_{3}\right)$. Since

$$
\frac{g(t)}{h(t)}=\frac{h\left(t_{2}\right)}{K\left(t_{2}, t_{3}\right)} \frac{\hat{g}(t)}{h(t)}
$$

and

$$
\left(\frac{\hat{g}(t)}{h(t)}\right)^{\prime}=\left(\frac{\lg ^{\frac{2}{p}} \frac{t_{3}}{t}}{\lg ^{\frac{2}{p}} t}\right)^{\prime}=\left[\left(\frac{\lg \frac{t_{3}}{t}}{\lg t}\right)^{\frac{2}{p}}\right]^{\prime}=-\frac{2}{p}\left(\frac{\lg \frac{t_{3}}{t}}{\lg t}\right)^{\frac{2-p}{p}} \frac{\lg t_{3}}{t \lg ^{2} t}<0,
$$

we see that $\left(\frac{g}{h}\right)^{\prime}<0$. Hence, $\frac{g}{h}$ is monotonic on $\left(t_{2}, t_{3}\right)$. Now, by the second mean value theorem of the integral calculus, there exists $\xi \in\left(t_{2}, t_{3}\right)$ such that

$$
\begin{aligned}
\int_{t_{2}}^{t_{3}}\left[c(t)-\frac{\gamma_{p}}{t^{p}}\right]|g(t)|^{p} d t & =\int_{t_{2}}^{t_{3}}\left[c(t)-\frac{\gamma_{p}}{t^{p}}\right]|h(t)|^{p}\left|\frac{g(t)}{h(t)}\right|^{p} d t \\
& =\int_{t_{2}}^{\xi}\left[c(t)-\frac{\gamma_{p}}{t^{p}}\right]|h(t)|^{p} .
\end{aligned}
$$

Consequently,

$$
\begin{aligned}
H\left(h ; t_{1}, t_{2}\right)+H\left(g ; t_{2}, t_{3}\right) & =\int_{t_{1}}^{t_{3}}\left(c(t)-\frac{\gamma(t)}{t^{p}}\right)|h(t)|^{p} d t \\
& =\int_{t_{1}}^{\xi}\left(c(t)-\frac{\gamma(t)}{t^{p}}\right)|h(t)|^{p} d t \\
& =\int_{t_{1}}^{\xi}\left(c(t)-\frac{\gamma(t)}{t^{p}}\right) t^{p-1} \lg ^{2} t d t .
\end{aligned}
$$


Let us now return to the computation of the functional $\mathscr{F}$.

$$
\begin{aligned}
\mathscr{F}\left(y ; t_{0}, t_{3}\right)= & L+G\left(h ; t_{1}, t_{2}\right)+G\left(g ; t_{2}, t_{3}\right)-\int_{t_{1}}^{\xi}\left(c(t)-\frac{\gamma_{p}}{t^{p}}\right) t^{p-1} \lg ^{2} t d t \\
& =\lg t_{2}\left[\frac{L}{\lg t_{2}}+2\left(\frac{p-1}{p}\right)^{p-1}-2\left(\frac{p-1}{p}\right)^{p-1} \frac{\lg t_{1}}{\lg t_{2}}+\left(\frac{p-1}{p}\right)^{p-1} \lg t_{2}\right. \\
& -\left(\frac{p-1}{p}\right)^{p-1} \frac{\lg ^{2} t_{1}}{\lg t_{2}}+o(1)+\frac{G\left(g ; t_{2}, t_{3}\right)}{\lg t_{2}} \\
& \left.-\frac{1}{\lg t_{2}} \int_{t_{1}}^{\xi}\left(c(t)-\frac{\gamma_{p}}{t^{p}}\right) t^{p-1} \lg ^{2} t d t\right] \\
& \leq \lg t_{2}\left[\frac{L}{\lg t_{2}}+2\left(\frac{p-1}{p}\right)^{p-1}+\left(\frac{p-1}{p}\right)^{p-1} \lg t_{2}+o(1)+\frac{G\left(g ; t_{2}, t_{3}\right)}{\lg t_{2}}\right. \\
& \left.-\frac{1}{\lg \xi} \int_{1}^{\xi}\left(c(t)-\frac{\gamma_{p}}{t^{p}}\right) t^{p-1} \lg ^{2} t d t+\frac{1}{\lg t_{2}} \int_{1}^{t_{1}}\left(c(t)-\frac{\gamma_{p}}{t^{p}}\right) t^{p-1} \lg ^{2} t d t\right] .
\end{aligned}
$$

Now, let $\varepsilon>0$ be such that the lower limit in (12) is greater than $2\left(\frac{p-1}{p}\right)^{p-1}+6 \varepsilon$ and let $t_{1}>t_{0}$ be arbitrary. We can choose $t_{2}>t_{1}$ in such a way that $\frac{L}{\lg t_{2}}<\varepsilon$, the term $o(1)$ is $<\varepsilon$ and

$$
\frac{1}{\lg \xi} \int_{1}^{\xi}\left(c(s)-\frac{\gamma_{p}}{s^{p}}\right) s^{p-1} \lg ^{2} s d s>2\left(\frac{p-1}{p}\right)^{p-1}+5 \varepsilon \quad \text { whenever } \xi>t_{2} .
$$

Finally, according to (15), we take $t_{3}>t_{2}$ such that

$$
\frac{G\left(g ; t_{2}, t_{3}\right)}{\lg t_{2}}<-\left(\frac{p-1}{p}\right)^{p-1} \lg t_{2}+\varepsilon
$$

Summarizing all the estimates above, we have

$$
\begin{aligned}
\mathscr{F}\left(y ; t_{0}, t_{3}\right) \leq & \lg t_{2}\left[\varepsilon+2\left(\frac{p-1}{p}\right)^{p-1}+\left(\frac{p-1}{p}\right)^{p-1} \lg t_{2}+\varepsilon\right. \\
& \left.-\left(\frac{p-1}{p}\right)^{p-1} \lg t_{2}+\varepsilon-2\left(\frac{p-1}{p}\right)^{p-1}-5 \varepsilon+\varepsilon\right]=-\varepsilon<0
\end{aligned}
$$

and, by Lemma 1, equation (1) is oscillatory.

\section{REMARKS AND COMMENTS}

In this concluding section, we formulate some open problems and suggestions for the further investigation of problems similar to the one solved in Theorem 1. 


\section{1}

Corollary 1 in [2] states that (1) is oscillatory if (6) holds, i. e., the constant $\frac{1}{2}\left(\frac{p-1}{p}\right)^{p-1}$ is 4 times less than the constant $2\left(\frac{p-1}{p}\right)^{p-1}$ in (12). This is a typical phenomenon of oscillation theory. Oscillation criteria proved by using the variational principle contain the oscillation constant which is 4-times larger than the constant in the same criterion but proved by the Riccati technique. Based on this observation, we conjecture that the constant $2\left(\frac{p-1}{p}\right)^{p-1}$ in (12) can be replaced by the constant $\frac{1}{2}\left(\frac{p-1}{p}\right)^{p-1}$ and the statement of Theorem 1 remains valid. The proof of this modified statement of Theorem 1 via the Riccati technique is the subject of the present investigation.

\section{2}

Corollary 2 in [2] gives the nonoscillation counterpart of the above mentioned Corollary 1 of that paper: Equation (1) is nonoscillatory provided (7) and (8) hold. We conjecture that a similar nonoscillation counterpart can be formulated also for Theorem 1 as follows.

Conjecture 1. Suppose that

$$
\limsup _{t \rightarrow \infty} \frac{1}{\lg t} \int_{t}^{\infty}\left(c(s)-\frac{\gamma_{p}}{s^{p}}\right) s^{p-1} \lg ^{2} s d s<\frac{1}{2}\left(\frac{p-1}{p}\right)^{p-1}
$$

and

$$
\liminf _{t \rightarrow \infty} \frac{1}{\lg t} \int_{t}^{\infty}\left(c(s)-\frac{\gamma_{p}}{s^{p}}\right) s^{p-1} \lg ^{2} s d s>-\frac{3}{2}\left(\frac{p-1}{p}\right)^{p-1} .
$$

Then (1) is nonoscillatory.

\section{3}

In oscillation and nonoscillation criteria of the above-mentioned papers $[\mathbf{2}, \mathbf{5}]$, where the half-linear equation

$$
\left(r(t) \Phi\left(x^{\prime}\right)\right)^{\prime}+c(t) \Phi(x)=0
$$

is viewed as a perturbation of a nonoscillatory equation

$$
\left(r(t) \Phi\left(x^{\prime}\right)\right)^{\prime}+\tilde{c}(t) \Phi(x)=0,
$$

a function $h$ appeared which is a solution of (16) (in case $r(t) \equiv 1$ and $\tilde{c}(t)=\frac{\gamma_{p}}{t^{p}}$ it is $h(t)=t^{\frac{p-1}{p}}$. In our main result, Theorem 1 , the function

$$
h(t)=t^{\frac{p-1}{p}} \lg ^{\frac{2}{p}} t
$$

is not a solution of (4) (equation (1) is viewed as a perturbation of this Euler equation), but it is only close to a solution of this equation, in a certain sense. This 
observation suggests another direction for the next investigation of (16) as a perturbation of (17)— to look for (non)oscillation criteria where $\int^{t}(c(s)-\tilde{c}(s))|h(s)|^{p} d s$ or $\int_{t}^{\infty}(c(s)-\tilde{c}(s))|h(s)|^{p} d s$ appear and $h$ generally is not a solution of (17) but it is only close to a solution of (17), in a certain sense.

\section{REFERENCES}

[1] DošLÝ, O.: Oscillation criteria for half-linear differential equations, Hiroshima J. Math., 28 (1998), 507-521.

[2] DošLÝ, O. and Lomtatidze, A.: Oscillation and nonoscillation criteria for half-linear second order differential equations, submitted.

[3] Elbert, Á.: Asymptotic behaviour of autonomous half-linear differential systems on the plane, Studia Sci. Math. Hungar., 19 (1984), 447-464.

[4] ElBERT, Á.: Oscillation and nonoscillation theorems for some non-linear ordinary differential equations, Lecture Notes in Math. No. 964 (1982), 187-212.

[5] Elbert, Á. And Schneider, A.: Perturbations of the half-linear Euler differential equation, Result. Math., 37 (2000), 56-83.

[6] Li, H. J. AND Yен, C. C.: Sturmian comparison theorem for half-linear second order differential equations, Proc. Roy. Soc. Edinburgh, 125A (1996), 1193-1204.

\section{Author's Address}

Jana ̌̌ezníčková:

Masaryk University, Department of Mathematics, JanáčKovo nám 2a, CZ-662 95 Brno, Czech REPUBLIC

E-mail address: janar@math.muni.cz 